\title{
Electronic structure of thallium filled skutterudites studied by $x$-ray absorption and Mössbauer spectroscopy
}

\author{
Gary J. Longa) \\ Department of Chemistry, University of Missouri-Rolla, Rolla, Missouri 65409-010 \\ Bernard Mahieu \\ Department of Chemistry, CSTR, Université Catholique de Louvain, Place L. Pasteur, 1, \\ B-1348 Louvain-la-Neuve, Belgium \\ Brian C. Sales \\ Solid State Division, Oak Ridge National Laboratory, Oak Ridge, Tennessee 37831 \\ Raphaël P. Hermann and Fernande Grandjean ${ }^{\text {b) }}$ \\ Department of Physics, B5, University of Liège, B-4000 Sart-Tilman, Belgium
}

(Received 6 September 2002; accepted 6 October 2002)

\begin{abstract}
Thallium $L_{\mathrm{III}}$-edge x-ray absorption and iron-57 and tin-119 Mössbauer spectral measurements have been carried out to probe the electronic structure of the three sites in the filled skutterudite $\mathrm{Tl}_{x} \mathrm{Co}_{4-y} \mathrm{Fe}_{y} \mathrm{Sb}_{12}$ and $\mathrm{Tl}_{x} \mathrm{Co}_{4} \mathrm{Sb}_{12-z} \mathrm{Sn}_{z}$ compounds with a range of $x, y$, and $z$ values. The thallium $L_{\mathrm{III}}$-edge x-ray absorption spectra are independent of $x, y$, and $z$ and are well reproduced by full multiple scattering calculations with a cluster with a radius of $8.5 \AA$ centered on thallium. The iron-57 Mössbauer spectra consist of two doublets and the major component is assigned to iron on the cobalt sublattice in view of its hyperfine parameters. The origin of the minor component is uncertain and is tentatively assigned to either iron with thallium vacancies as next nearest neighbors or to iron in the voids. The tin-119 Mössbauer spectral hyperfine parameters clearly indicate that tin substitutes for antimony in the skutterudite structure. Further, the tin electronic configuration is very similar to that of antimony and is insensitive to the thallium content. (C) 2002 American Institute of Physics. [DOI: 10.1063/1.1525069]
\end{abstract}

\section{INTRODUCTION}

The interesting and varied physical properties of the filled skutterudite family of compounds have, over the past ten years, prompted both theoretical ${ }^{1-4}$ and experimental ${ }^{5-8}$ work. Among these physical properties, the semiconducting ${ }^{5-7,9,10}$ behavior and the low-thermal conductivity, ${ }^{6,7,10-13}$ particularly of the rare-earth filled skutterudites, make them potential thermoelectric materials with many technological and commercial applications. The useful electronic and dynamic properties of the filled skutterudites, whose structure is based on that of $\mathrm{CoSb}_{3}$, is the result of the bonding of the atoms within this open framework structure. Thus, any improvement in their technologically important properties will proceed from a better understanding of the electronic structure of the different atoms within the filled skutterudites. Our previous studies ${ }^{14-16}$ of $\mathrm{Ce}_{x} \mathrm{Co}_{4-y} \mathrm{Fe}_{y} \mathrm{Sb}_{12}$ and $\mathrm{Yb}_{x} \mathrm{Co}_{4} \mathrm{Sb}_{12-z} \mathrm{Sn}_{z}$ by both x-ray absorption and iron-57 and tin-119 Mössbauer spectroscopy, have characterized the electronic ground-state configuration of the rare-earth atom and the electronic structure of the iron and tin atoms.

More recently, the filling of the voids in the skutterudite structure with thallium ${ }^{17}$ has been achieved. Because thallium has a formal valence of +1 , iron or tin must replace some of the cobalt or antimony, respectively, to maintain

\footnotetext{
a) Electronic mail: glong@umr.edu

${ }^{\mathrm{b})}$ Electronic mail: fgrandjean@ulg.ac.be
}

charge neutrality. As a consequence, two series of thallium filled skutterudites, $\mathrm{Tl}_{x} \mathrm{Co}_{4-y} \mathrm{Fe}_{y} \mathrm{Sb}_{12}$ and $\mathrm{Tl}_{x} \mathrm{Co}_{4} \mathrm{Sb}_{12-z} \mathrm{Sn}_{z}$, have been prepared and characterized. ${ }^{17}$ Exceptionally large atomic mean-square displacements have been observed for the thallium filling atoms and their "rattling" helps produce the large thermal resistivity observed for these filled skutterudites. Whereas the macroscopic properties of these two series of compounds have been carefully measured, ${ }^{17}$ their microscopic properties have not been investigated in detail. Hence, with the goal of elucidating the electronic structure of thallium, iron, and tin, we have carried out a thallium $L_{\mathrm{III}}$-edge x-ray absorption study of these two series of compounds and an iron-57 and tin-119 Mössbauer spectral study of $\mathrm{Tl}_{x} \mathrm{Co}_{4-y} \mathrm{Fe}_{y} \mathrm{Sb}_{12}$ and $\mathrm{Tl}_{x} \mathrm{Co}_{4} \mathrm{Sb}_{12-z} \mathrm{Sn}_{z}$, respectively.

\section{EXPERIMENT}

The samples studied herein are the same as those previously synthesized. ${ }^{17}$ The thallium $L_{\mathrm{III}}$-edge x-ray absorption near-edge structure (XANES) spectra were obtained at $295 \mathrm{~K}$ on beamline 4-3 at the Stanford Synchrotron Radiation Laboratory, Stanford, California. The measurements were carried out in transmission mode with a $\operatorname{Si}(111) \phi=90^{\circ}$ double-crystal monochromator using ion chambers filled with a mixture of nitrogen and helium and placed both in front and behind the sample. Harmonic contamination was minimized by detuning the monochromator. The spectra were recorded between 12.60 and $12.64 \mathrm{keV}$ with a step of $10 \mathrm{eV}$ and between 12.64 and $12.80 \mathrm{keV}$ with a step of 0.35 
$\mathrm{eV}$. The $K$-edge absorption spectrum of a thin lead foil was used for energy calibration. The absorbance of the XANES spectra have been normalized at the highest absorption peak.

The iron-57 Mössbauer spectra of $\mathrm{Tl}_{0.8} \mathrm{Co}_{3} \mathrm{FeSb}_{12}$ and $\mathrm{Tl}_{0.5} \mathrm{Co}_{3.5} \mathrm{Fe}_{0.5} \mathrm{Sb}_{12}$ were measured at 90 and $295 \mathrm{~K}$ on a constant-acceleration spectrometer which was calibrated at room temperature with $\alpha$-iron foil. The iron-57 Mössbauer spectra were obtained with a room-temperature cobalt-57 rhodium matrix source and the isomer shifts are reported relative to room-temperature $\alpha$-iron foil. The absorber thicknesses were $\sim 30 \mathrm{mg} / \mathrm{cm}^{2}$ and the samples were dispersed in boron nitride. The spectra were fit as discussed in Sec. IV and the resulting isomer shifts, quadrupole splittings, and linewidths are accurate to $\pm 0.005 \mathrm{~mm} / \mathrm{s}$.

The tin-119 Mössbauer spectra of $\mathrm{Tl}_{0.5} \mathrm{Co}_{4} \mathrm{Sb}_{11.5} \mathrm{Sn}_{0.5}$, $\mathrm{Tl}_{0.95} \mathrm{Co}_{4} \mathrm{Sb}_{11.25} \mathrm{Sn}_{0.75}$, and $\mathrm{TlCo}_{4} \mathrm{Sb}_{11} \mathrm{Sn}$ were measured at $83 \mathrm{~K}$ on a constant-acceleration spectrometer which was calibrated at room temperature with $\alpha$-iron foil. The tin-119 spectra were obtained with a room-temperature tin- $119 m$ $\mathrm{CaSnO}_{3}$ matrix source and the isomer shifts are reported relative to room-temperature $\mathrm{CaSnO}_{3}$. The absorber thicknesses were $70 \mathrm{mg} / \mathrm{cm}^{2}$ and the samples were dispersed in boron nitride. The spectra were fit with a symmetric quadrupole doublet and the resulting isomer shifts, quadrupole splittings, and linewidths are accurate to $\pm 0.02 \mathrm{~mm} / \mathrm{s}$.

\section{THALLIUM $L_{\| I I}$-EDGE XANES SPECTRAL RESULTS}

The $295 \mathrm{~K}$ thallium $L_{\mathrm{III}}$-edge XANES spectra for several of the $\mathrm{Tl}_{x} \mathrm{Co}_{4-y} \mathrm{Fe}_{y} \mathrm{Sb}_{12}$ and $\mathrm{Tl}_{x} \mathrm{Co}_{4} \mathrm{Sb}_{12-z} \mathrm{Sn}_{z}$ compounds are shown in Fig. 1. To our knowledge, there are no reported XANES or extended $\mathrm{x}$-ray absorption fine structure studies of thallium metal or thallium compounds at the thallium $L_{\mathrm{III}}$ edge. Hence, no comparison of our results with previously published thallium spectra is possible.

It is obvious from Fig. 1 that there are virtually no differences between the XANES spectra of the different $\mathrm{Tl}_{x} \mathrm{Co}_{4-y} \mathrm{Fe}_{y} \mathrm{Sb}_{12}$ and $\mathrm{Tl}_{x} \mathrm{Co}_{4} \mathrm{Sb}_{12-z} \mathrm{Sn}_{z}$ compounds. This similarity can be understood in terms of the near-neighbor environment of thallium in the filled skutterudite structure. Indeed, for all the $\mathrm{Tl}_{x} \mathrm{Co}_{4-y} \mathrm{Fe}_{y} \mathrm{Sb}_{12}$ compounds, independent of $x$ and $y$, the first neighbors of thallium are the 12 antimony atoms at $\sim 3.5 \AA$. In $\mathrm{Tl}_{0.1} \mathrm{Co}_{4} \mathrm{Sb}_{12}$ and $\mathrm{Tl}_{0.22} \mathrm{Co}_{4} \mathrm{Sb}_{12}$ the second neighbors are eight cobalt atoms at $\sim 4 \AA$. In $\mathrm{Tl}_{0.5} \mathrm{Co}_{3.5} \mathrm{Fe}_{0.5} \mathrm{Sb}_{12}$ and $\mathrm{Tl}_{0.8} \mathrm{Co}_{3} \mathrm{FeSb}_{12}$, on average, one and two cobalt atoms, respectively, are replaced by iron atoms; a replacement which does not dramatically change the amplitude and phase of the backscattered photoelectron wave as compared to scattering by cobalt. For all compounds, the subsequent shells of neighbors contain 24 and 12 antimony atoms at $\sim 6$ and $8.5 \AA$ and, on average, 0.8 and 1.66 thallium atoms at $\sim 8 \AA$. In the $\mathrm{Tl}_{x} \mathrm{Co}_{4} \mathrm{Sb}_{12-z} \mathrm{Sn}_{z}$ compounds, a small fraction of at most $1 / 12$ of the antimony neighboring atoms are replaced by tin atoms, atoms which again do not substantially change the amplitude and phase of the backscattered photoelectron wave. Hence, the shape of the XANES spectra is produced by the scattering of the photoelectron wave by antimony and cobalt atoms, the positions of which vary according to the crystallographic parameters. ${ }^{17}$

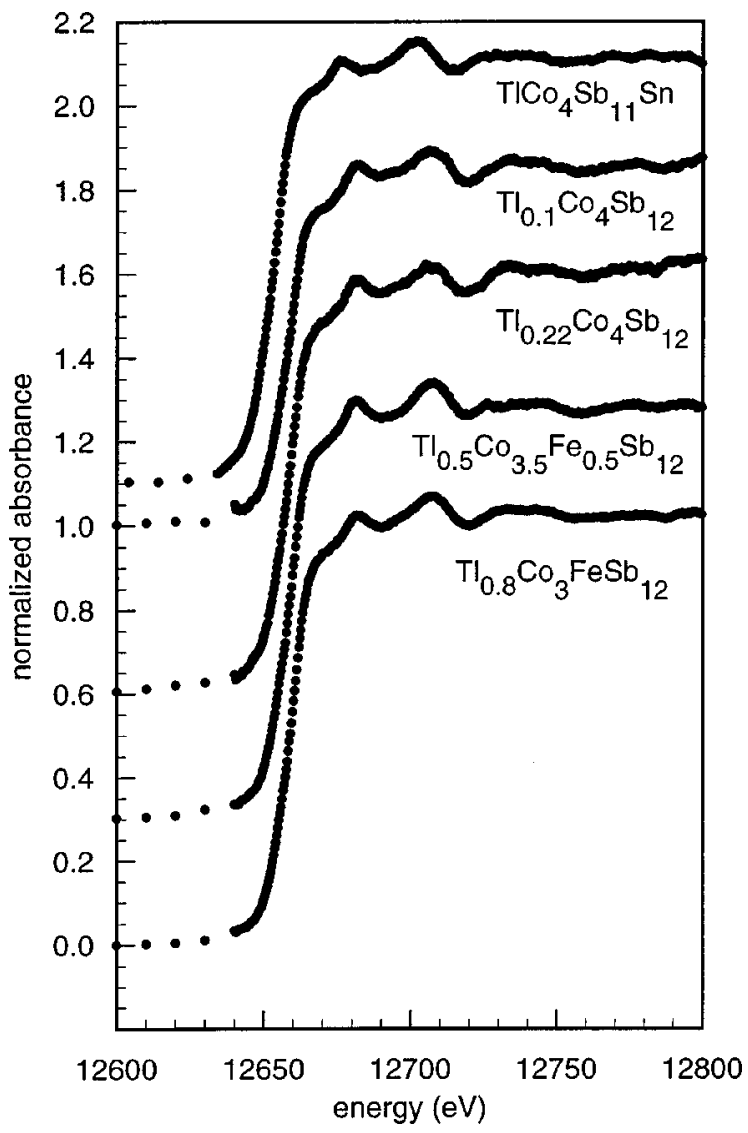

FIG. 1. The thallium $L_{\mathrm{III}}$-edge XANES spectra obtained at $295 \mathrm{~K}$ for the indicated compounds. The different spectra have been shifted vertically by an arbitrary amount for better presentation.

In order to confirm this interpretation, full multiple scattering calculations of the thallium $L_{\mathrm{III}}$-edge XANES spectrum for the hypothetical $\mathrm{TlCo}_{4} \mathrm{Sb}_{12}$ compound, with a cubic unit-cell $a$-lattice parameter of $9.109 \AA$ and antimony positional parameters of $y=0.3368$ and $z=0.1593$, have been carried $^{18,19}$ out with the FEFF8.10 code. No attempt was made to model any of the disorder resulting from the distribution of iron and cobalt atoms, in the first case, or antimony and tin atoms, in the second case. The calculated spectra obtained for clusters of various radii centered on thallium are shown in Fig. 2(a). The general shape of the calculated spectrum with the three peaks at $\sim 12665,12680$, and $12710 \mathrm{eV}$ is reproduced with a cluster containing only 12 antimony firstneighbor atoms, but the energies of the peaks are not accurately reproduced. More specifically, the calculated energy of the first peak is at a higher energy than the observed energy. Indeed, a large cluster of $8.5 \AA$ radius is necessary to reproduce the first peak in the rising edge at the observed energy.

A comparison of the experimental spectrum of $\mathrm{Tl}_{0.8} \mathrm{Co}_{3} \mathrm{FeSb}_{12}$ with the calculated spectrum for a cluster of $8.5 \AA$ radius in hypothetical $\mathrm{TlCo}_{4} \mathrm{Sb}_{12}$ is shown in Fig. 2(b). The major peaks in the experimental spectrum are well reproduced in the calculated spectrum with the exception of the calculated peak at $12690 \mathrm{eV}$, a peak which is not observed experimentally. However the intensity of this peak increases for the cluster of $8 \AA$, a cluster that includes thal- 

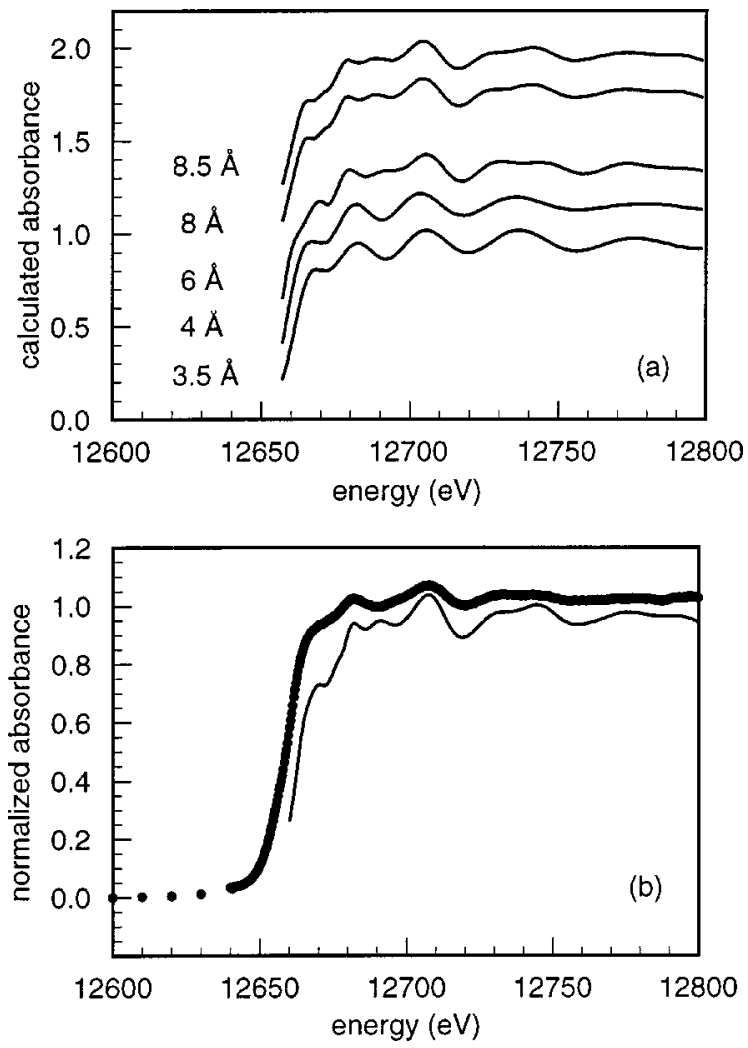

FIG. 2. (a) The thallium $L_{\mathrm{III}}$-edge XANES spectra calculated for the hypothetical $\mathrm{TlCo}_{4} \mathrm{Sb}_{12}$ compound by using the FEFF8.10 code for the clusters with the indicated radii. The different spectra have been shifted vertically by an arbitrary amount for a better display. (b) The experimental thallium $L_{\text {III }}$-edge XANES spectrum of $\mathrm{Tl}_{0.8} \mathrm{Co}_{3} \mathrm{FeSb}_{12}$ and the spectrum calculated for $\mathrm{TlCo}_{4} \mathrm{Sb}_{12}$ by using the FEFF8.10 code and a cluster of $8.5 \AA$ radius centered on thallium.

lium neighbor sites which are not fully occupied in the actual compound.

\section{IRON-57 MÖSSBAUER SPECTRAL RESULTS}

The iron-57 Mössbauer spectra of $\mathrm{Tl}_{0.8} \mathrm{Co}_{3} \mathrm{FeSb}_{12}$ and $\mathrm{Tl}_{0.5} \mathrm{Co}_{3.5} \mathrm{Fe}_{0.5} \mathrm{Sb}_{12}$ obtained at 90 and $295 \mathrm{~K}$ are shown in Fig. 3. These spectra have been fit with two symmetric Lorentzian quadrupole doublets, whose hyperfine parameters and relative areas are given in Table I. The major component is assigned to iron on the cobalt sublattice of the filled skutterudite. The identity of the minor component is less certain. Its hyperfine parameters do not correspond ${ }^{20}$ to either $\mathrm{FeSb}_{2}$ or $\mathrm{FeSb}$ and there are, to our knowledge, no known TlFe alloys. Further, no TlFe impurity was detected ${ }^{17}$ in either sample by $\mathrm{x}$ ray or neutron diffraction.

Within the skutterudite lattice, there are two possible assignments of the minor component observed in Fig. 3. First, because of the partial thallium filling, the iron atoms on the cobalt sublattice have either two, one, or zero thallium atoms as second neighbors. In $\mathrm{Tl}_{0.8} \mathrm{Co}_{3} \mathrm{FeSb}_{12}$, the probabilities of having two, one, and zero thallium neighbors are $0.64,0.32$, and 0.04 , and in $\mathrm{Tl}_{0.5} \mathrm{Co}_{3.5} \mathrm{Fe}_{0.5} \mathrm{Sb}_{12}$, the probabilities are $0.25,0.50$, and 0.25 . Hence, it is difficult to correlate the relative absorption area of $\sim 15 \%$ observed in

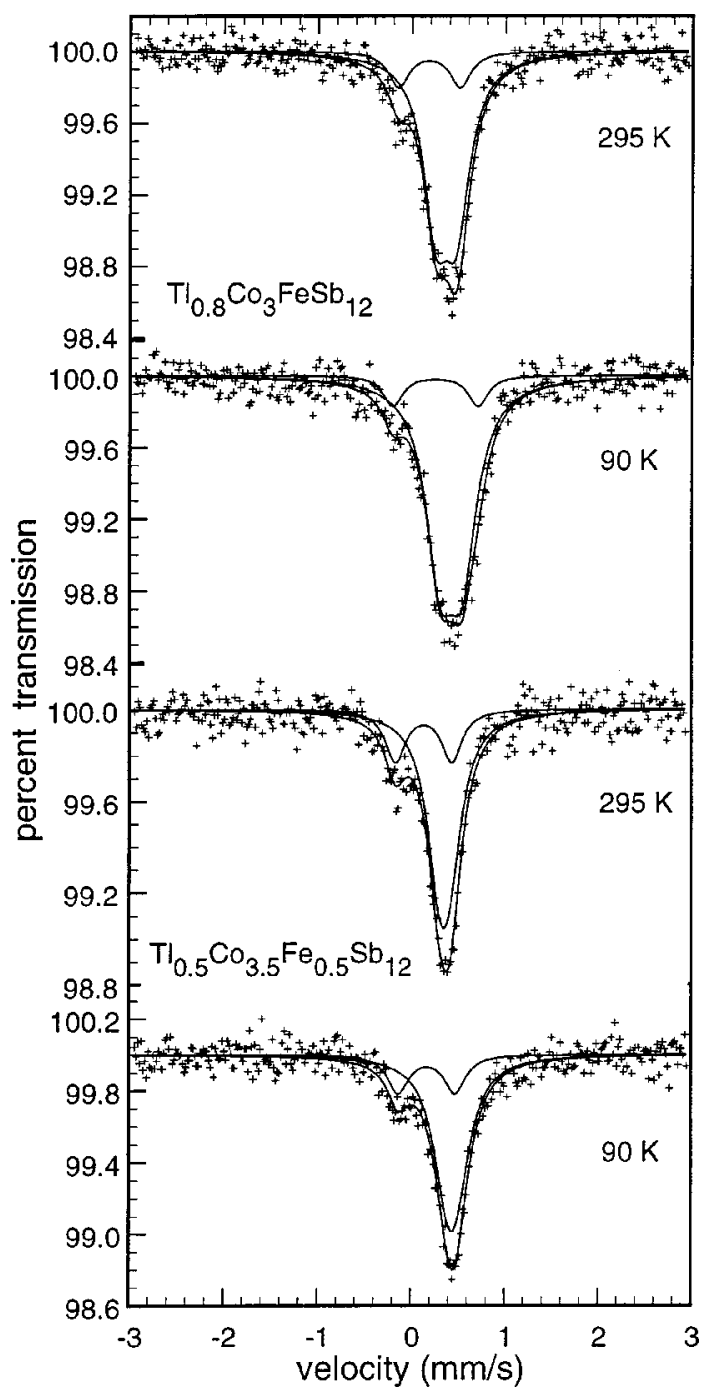

FIG. 3. The iron-57 Mössbauer spectra of $\mathrm{Tl}_{0.8} \mathrm{Co}_{3} \mathrm{FeSb}_{12}$ and $\mathrm{Tl}_{0.5} \mathrm{Co}_{3.5} \mathrm{Fe}_{0.5} \mathrm{Sb}_{12}$ obtained at 90 and $295 \mathrm{~K}$.

$\mathrm{Tl}_{0.8} \mathrm{Co}_{3} \mathrm{FeSb}_{12}$ and of $\sim 25 \%$ observed in $\mathrm{Tl}_{0.5} \mathrm{Co}_{3.5} \mathrm{Fe}_{0.5} \mathrm{Sb}_{12}$ with these probabilities.

Of course, in contrast with cerium, ${ }^{11}$ the thallium vacancies may prefer to associate with iron or, alternatively, the thallium atoms may prefer to form a pair around either iron or cobalt. In either of these cases, the thallium does not randomly fill the voids and, as a consequence, the minor doublet could be assigned to iron on the cobalt sublattice with thallium vacancies as second neighbors. In addition, our unpublished spectra of $\mathrm{Fe}_{y} \mathrm{Co}_{1-\gamma} \mathrm{Sb}_{3}$ with $y$ between 0.05 and 0.2 show, at $85 \mathrm{~K}$, a doublet with an isomer shift of $0.44 \mathrm{~mm} / \mathrm{s}$ and a quadrupole splitting of $0.72 \mathrm{~mm} / \mathrm{s}$, values which are similar to those of the minor doublet, a similarity that would support the above assignment of this doublet. Second, the minor doublet may be assigned to iron filling the voids of the skutterudite lattice.

The iron-57 quadrupole splitting observed ${ }^{14,21,22}$ for various filled skutterudites vary from 0.14 to $0.44 \mathrm{~mm} / \mathrm{s}$. The existence of a quadrupole splitting at the iron site indicates that there is a distortion from cubic symmetry at the $8 c$ crystallographic site. The filled skutterudite has a cubic structure 
TABLE I. Iron-57 Mössbauer spectral hyperfine parameters.

\begin{tabular}{lccccll}
\hline \hline Compound & $\begin{array}{c}T \\
(\mathrm{~K})\end{array}$ & $\begin{array}{c}\delta \\
(\mathrm{mm} / \mathrm{s})\end{array}$ & $\begin{array}{c}\Delta E_{Q} \\
(\mathrm{~mm} / \mathrm{s})\end{array}$ & $\begin{array}{c}\Gamma \\
(\mathrm{mm} / \mathrm{s})\end{array}$ & $\begin{array}{c}\text { Area } \\
(\%)\end{array}$ & \multicolumn{1}{c}{ Assignment } \\
\hline $\mathrm{Tl}_{0.8} \mathrm{Co}_{3} \mathrm{FeSb}_{12}$ & 295 & 0.36 & 0.22 & 0.34 & 84 & $\mathrm{Fe}$ on the Co sublattice \\
& & 0.18 & 0.66 & 0.27 & 16 & uncertain \\
& 90 & 0.42 & 0.24 & 0.37 & 89 & $\begin{array}{l}\text { Fe on the Co sublattice } \\
\text { uncertain }\end{array}$ \\
& & 0.25 & 0.91 & 0.26 & 11 & \\
$\mathrm{Tl}_{0.5} \mathrm{Co}_{3.5} \mathrm{Fe}_{0.5} \mathrm{Sb}_{12}$ & 295 & 0.35 & 0.11 & 0.33 & 75 & $\mathrm{Fe}$ on the Co sublattice \\
& & 0.13 & 0.60 & 0.26 & 25 & uncertain \\
& 90 & 0.44 & 0.10 & 0.36 & 77 & Fe on the Co sublattice \\
& & 0.17 & 0.60 & 0.28 & 23 & uncertain \\
\hline \hline
\end{tabular}

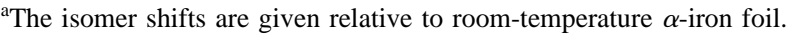

in which the distortion from a perfect square of the $\mathrm{Sb}_{4}$ ring has been used as a measure of its degree of distortion. The correlation between the quadrupole splitting and the distortion of the $\mathrm{Sb}_{4}$ square is shown in Fig. 4 for the filled skutterudites with the indicated filling atom. In Fig. 4, the distortion of the antimony square is given as the difference in length between two edges of the "square" or better the rectangle of antimony atoms and the quadrupole splitting is that observed for the maximum filling of the skutterudite compound. Whereas there is a fair correlation for the skutterudites in which the filling atom has a formal valence between +2 and +3 , the quadrupole splitting measured for $\mathrm{Tl}_{0.8} \mathrm{Co}_{3} \mathrm{FeSb}_{12}$ is substantially smaller than expected from the distortion of the antimony $\mathrm{Sb}_{4}$ square. In the case of the $\mathrm{Ce}_{x} \mathrm{Co}_{4-y} \mathrm{Fe}_{y} \mathrm{Sb}_{12}$ compounds, ${ }^{14}$ the lattice and conductionelectron contributions to the quadrupole splitting were estimated to be $\sim 0.16$ and $0.25 \mathrm{~mm} / \mathrm{s}$, respectively. Hence, if the lattice contribution to the quadrupole splitting in $\mathrm{Tl}_{0.8} \mathrm{Co}_{3} \mathrm{FeSb}_{12}$ is assumed to be $0.16 \mathrm{~mm} / \mathrm{s}$, then the conduction-electron contribution is only $\sim 0.06 \mathrm{~mm} / \mathrm{s}$. The different conduction-electron contributions may result from the $p$-type and $n$-type conduction mechanisms in the

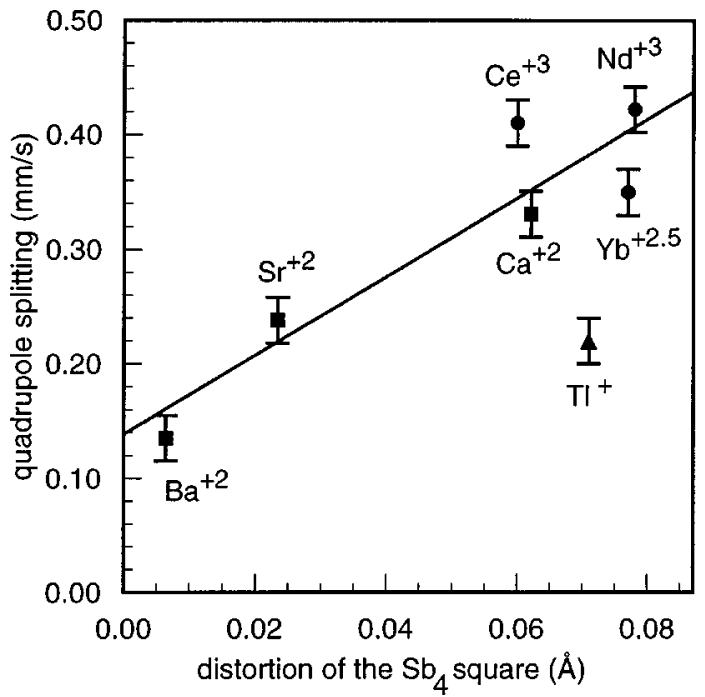

FIG. 4. The correlation between the $295 \mathrm{~K}$ iron- 57 quadrupole splitting in the filled skutterudites for the indicated filling atom and the distortion of the $24 g$ site antimony $\mathrm{Sb}_{4}$ rectangles.
$\mathrm{Ce}_{x} \mathrm{Co}_{4-y} \mathrm{Fe}_{y} \mathrm{Sb}_{12}$ and $\mathrm{Tl}_{x} \mathrm{Co}_{4-y} \mathrm{Fe}_{y} \mathrm{Sb}_{12}$ and $\mathrm{Tl}_{x} \mathrm{Co}_{4} \mathrm{Sb}_{12-z} \mathrm{Sn}_{z}$ compounds, respectively.

The correlation between the iron-57 isomer shift and the Wigner-Seitz cell volume available to the iron atoms in the $R_{2} \mathrm{Fe}_{17}$ and $R \mathrm{Fe}_{11} \mathrm{Ti}$ compounds and their hydrides and nitrides have proven ${ }^{23,24}$ very successful in understanding the electronic structure of these compounds. This correlation is shown in Fig. 5 for the same filled skutterudites included in Fig. 4. The Wigner-Seitz cell volumes have been calculated ${ }^{25}$ from crystallographic parameters from literature and by using the 12-coordinate metallic radii of the different atoms. The good linear correlation shown in Fig. 5 indicates that the $s$-electron density at the iron nucleus decreases and, hence, the isomer shift increases with increases in the Wigner-Seitz cell volume of the iron site. It is rather remarkable that the Tl skutterudite, for which the iron WignerSeitz cell volume is substantially smaller than those observed in the other skutterudites, does indeed show a substantially smaller isomer shift. In conclusion, the variation of the isomer shift in the filled skutterudites can be understood in terms of the influence of the site size on the s-electron density at the iron nucleus.

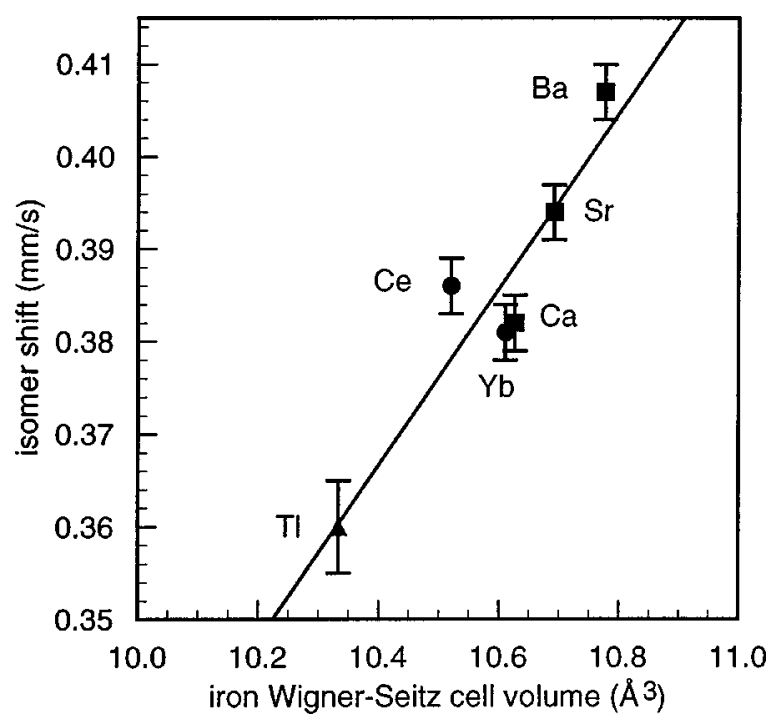

FIG. 5. The correlation between the $295 \mathrm{~K}$ iron-57 isomer shift in the filled skutterudites for the indicated filling atom and the iron Wigner-Seitz cell volume. 


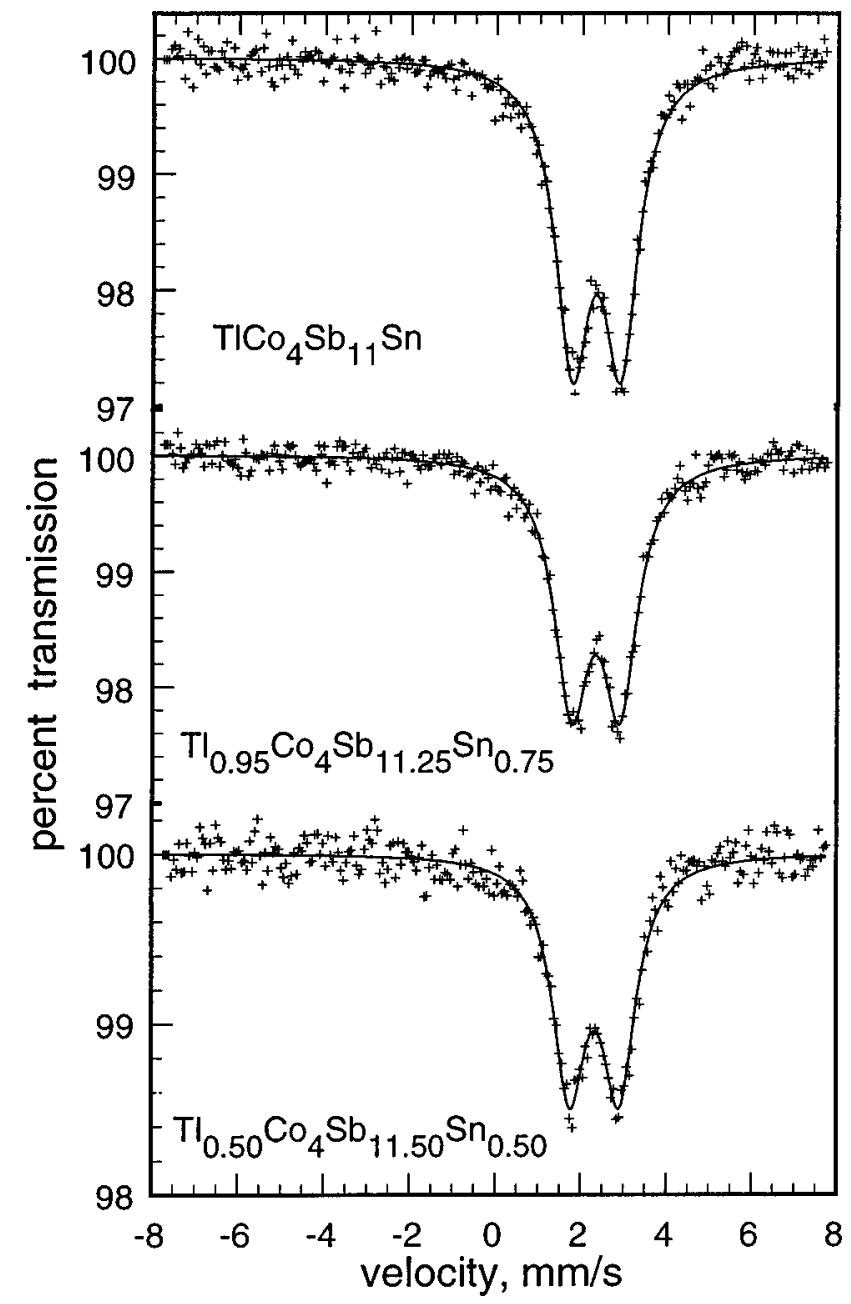

FIG. 6. The tin-119 Mössbauer spectra of $\mathrm{TlCo}_{4} \mathrm{Sb}_{11} \mathrm{Sn}$, $\mathrm{Tl}_{0.95} \mathrm{Co}_{4} \mathrm{Sb}_{11.25} \mathrm{Sn}_{0.75}$, and $\mathrm{Tl}_{0.5} \mathrm{Co}_{4} \mathrm{Sb}_{11.5} \mathrm{Sn}_{0.5}$ obtained at $83 \mathrm{~K}$.

\section{TIN-119 MÖSSBAUER SPECTRAL RESULTS}

The tin-119 Mössbauer spectra of $\mathrm{TlCo}_{4} \mathrm{Sb}_{11} \mathrm{Sn}$, $\mathrm{Tl}_{0.95} \mathrm{Co}_{4} \mathrm{Sb}_{11.25} \mathrm{Sn}_{0.75}$, and $\mathrm{Tl}_{0.5} \mathrm{Co}_{4} \mathrm{Sb}_{11.5} \mathrm{Sn}_{0.5}$ obtained at 83 $\mathrm{K}$ are shown in Fig. 6 and the corresponding hyperfine parameters are given in Table II. A comparison of the $\mathrm{Tl}_{x} \mathrm{Co}_{4} \mathrm{Sb}_{12-z} \mathrm{Sn}_{z}$ spectra with those of related tin-containing skutterudites ${ }^{26}$ clearly indicates that the observed doublet must be assigned to tin which resides on the antimony sublattice of the basic $\mathrm{CoSb}_{3}$ skutterudite structure. The observed isomer shift of ca. $2.3 \mathrm{~mm} / \mathrm{s}$ is typical of tin in an intermetallic compound and, as expected for the open frame-

TABLE II. Tin-119 Mössbauer spectral hyperfine parameters obtained at 83 K.

\begin{tabular}{lcccc}
\hline \hline Compound & $\begin{array}{c}\delta \\
(\mathrm{mm} / \mathrm{s})^{\mathrm{a}}\end{array}$ & $\begin{array}{c}\Delta E_{Q} \\
(\mathrm{~mm} / \mathrm{s})\end{array}$ & $\begin{array}{c}\Gamma \\
(\mathrm{mm} / \mathrm{s})\end{array}$ & $\begin{array}{c}\text { Area } \\
(\%)\end{array}$ \\
\hline $\mathrm{TlCo}_{4} \mathrm{Sb}_{11} \mathrm{Sn}$ & 2.34 & 1.14 & 0.97 & 100 \\
$\mathrm{Tl}_{0.95} \mathrm{Co}_{4} \mathrm{Sb}_{11.25} \mathrm{Sn}_{0.75}$ & 2.34 & 1.14 & 0.99 & 100 \\
$\mathrm{Tl}_{0.50} \mathrm{Co}_{4} \mathrm{Sb}_{11.50} \mathrm{Sn}_{0.50}$ & 2.33 & 1.17 & 0.95 & 100 \\
\hline
\end{tabular}

${ }^{\mathrm{a}}$ The isomer shifts are given relative to room-temperature $\mathrm{CaSnO}_{3}$. work structure of $\mathrm{CoSb}_{3}$, its isomer shift and, hence, the $s$-electron density at the tin-119 nucleus is smaller than that observed in $\beta$-tin.

The tin-119 isomer shift follows a simple function ${ }^{27}$ of the effective number of $5 s$ and $5 p$ electrons given by

$$
\delta=-0.38+3.10 n_{s}-0.20 n_{s}^{2}-0.17 n_{s} n_{p},
$$

where $\delta$ is the isomer shift referred to $\mathrm{CaSnO}_{3}$ and $n_{s}$ and $n_{p}$ are the effective number of $5 s$ and $5 p$ electrons, respectively. This equation indicates that the electronic configuration of tin in the filled skutterudites is between $5 s^{0.9} 5 p^{0}$ and $5 s^{1.15} 5 p^{3}$. For comparison, the antimony- 121 isomer shift $\mathrm{t}^{28}$ in $\mathrm{CoSb}_{3}$ is more negative than in InSb, in which antimony is $5 s^{1} 5 p^{3}$. Hence, the $5 s$ electron density at the antimony nucleus is higher in $\mathrm{CoSb}_{3}$ and antimony has an electronic configuration between $5 s^{1+x} 5 p^{3}$ and $5 s^{1} 5 p^{3-x}$. Electronic band structure calculations ${ }^{29}$ give a $5 s$ population of 1.77 , a value which is in good agreement with this interpretation of the antimony isomer shift. In conclusion, the tin and antimony on the $24 \mathrm{~g}$ site of the filled skutterudite structure have very similar electronic configurations.

As may be observed in Fig. 6, the low symmetry at the antimony site in the skutterudite structure leads to a substantial quadrupole splitting of $\sim 1.15 \mathrm{~mm} / \mathrm{s}$ for the tin substituted onto the $24 g$ site. The antimony-121 Mössbauer spectrum of unfilled skutterudite, $\mathrm{CoSb}_{3}$, reported by Kjekshus et $a l .{ }^{28}$ exhibited a quadrupole interaction of $9 \mathrm{~mm} / \mathrm{s}$ and an asymmetry parameter, $\eta$, of 1.0. From this quadrupole interaction and by using a ground-state nuclear quadrupole moment $^{30}$ of 0.28 barn and $R_{Q}=1.34$ for antimony-121, an electric-field gradient at the antimony site of 3.0 $\times 10^{22} \mathrm{~V} / \mathrm{m}^{2}$ is obtained. From the $\sim 1.15 \mathrm{~mm} / \mathrm{s}$ quadrupole splitting measured herein and by using a nuclear quadrupole moment $^{31}$ of 0.064 barn for the tin- $119 m$ excited state, an electric-field gradient at the tin site of $2.8 \times 10^{22} \mathrm{~V} / \mathrm{m}^{2}$ is obtained. This close to perfect agreement provides excellent support for the assignment of this quadrupole doublet to tin which replaces antimony in the skutterudite structure.

The similarity of the hyperfine parameters given in Table II, indicates that the electronic properties of the rather isolated tin atoms are independent of the thallium and tin contents. This is reasonable because tin on the antimony $24 g$ site has only one thallium near neighbor at a distance of $\sim 3.4 \AA$ in the fully filled skutterudite.

\section{CONCLUSIONS}

The microscopic properties of the thallium, iron, and tin atoms in the $\mathrm{Tl}_{x} \mathrm{Co}_{4-y} \mathrm{Fe}_{y} \mathrm{Sb}_{12}$ and $\mathrm{Tl}_{x} \mathrm{Co}_{4} \mathrm{Sb}_{12-z} \mathrm{Sn}_{z}$ filled skutterudites have been probed by thallium $L_{\mathrm{III}}$-edge x-ray absorption and iron-57 and tin-119 Mössbauer spectroscopy. The thallium $L_{\mathrm{III}}$-edge $\mathrm{x}$-ray absorption spectra are well modeled by full multiple scattering calculations, a scattering which occurs within a cluster of $8.5 \AA$ radius centered on the thallium atom. The iron-57 Mössbauer spectra show two components. The major doublet is similar to that observed $^{14,16}$ in the other rare-earth filled skutterudites and is assigned to iron on the cobalt sublattice. The origin of the minor doublet is uncertain. On the basis of its hyperfine pa- 
rameters it is tentatively assigned to either iron on the cobalt sublattice with thallium vacancies as next nearest neighbors or to iron in the voids. The tin-119 Mössbauer spectra show one doublet, which is assigned without doubt to tin on the antimony sublattice. On the basis of the isomer shift, the electronic configuration of tin in the filled skutterudites is between $5 s^{0.9} 5 p^{0}$ and $5 s^{1.15} 5 p^{3}$ and is similar to that of antimony.

\section{ACKNOWLEDGMENTS}

The authors acknowledge with thanks the assistance of Dr. J. Y. Raty of the University of Liège and Drs. M. Latimer, S. George, N. George, and M. George, and the very helpful staff of the Stanford Synchrotron Radiation Laboratory. This work was supported in part by the "Fonds National de la Recherche Scientifique," Brussels, Belgium, Grant No. 9.456595.

${ }^{1}$ D. J. Singh and W. E. Pickett, Phys. Rev. B 50, 11235 (1994).

${ }^{2}$ L. Nordström and D. J. Singh, Phys. Rev. B 53, 1103 (1996).

${ }^{3}$ J. L. Feldman and D. J. Singh, Phys. Rev. B 53, 6273 (1996).

${ }^{4}$ D. J. Singh, I. I. Mazin, J. L. Feldman, and M. Fornari, Mater. Res. Soc. Symp. Proc. 545, 3 (1999).

${ }^{5}$ D. T. Morelli, T. Caillat, J. P. Fleurial, A. Borshchevsky, J. Vandersande, B. Chen, and C. Uher, Phys. Rev. B 51, 9622 (1995).

${ }^{6}$ D. T. Morelli and G. P. Meisner, J. Appl. Phys. 77, 3777 (1995).

${ }^{7}$ B. Chen, J. H. Xu, C. Uher, D. T. Morelli, G. P. Meisner, J. P. Fleurial, T. Caillat, and A. Borshchevsky, Phys. Rev. B 55, 1476 (1997).

${ }^{8}$ J. P. Fleurial, A. Borshchevsky, T. Caillat, D. T. Morelli, and G. P. Meisner, in Fifteenth International Conference on Thermoelectrics, (IEEE, New York, 1996), p. 91.

${ }^{9}$ H. Anno, K. Hatada, H. Shimizu, K. Matsubara, Y. Notohara, T. Sakakibara, H. Tashiro, and K. Motoya, J. Appl. Phys. 83, 5270 (1998).

${ }^{10}$ N. R. Dilley, E. D. Bauer, M. B. Maple, S. Dordevic, D. N. Basov, F. Freibert, T. W. Darling, A. Migliori, B. C. Chakoumakos, and B. C. Sales, Phys. Rev. B 61, 4608 (2000).
${ }^{11}$ G. P. Meisner, D. T. Morelli, S. Hu, J. Yang, and C. Uher, Phys. Rev. Lett. 80, 3551 (1998).

${ }^{12}$ B. C. Sales, B. C. Chakoumakos, D. Mandrus, J. W. Sharp, N. R. Dilley, and M. B. Maple, Mater. Res. Soc. Symp. Proc. 545, 13 (1999).

${ }^{13}$ B. C. Sales, B. C. Chakoumakos, and D. Mandrus, Mater. Res. Soc. Symp. Proc. 626, Z7.1.1 (2000).

${ }^{14}$ G. J. Long, D. Hautot, F. Grandjean, D. T. Morelli, and G. P. Meisner, Phys. Rev. B 60, 7410 (1999).

${ }^{15}$ F. Grandjean, G. J. Long, R. Cortes, D. T. Morelli, and G. P. Meisner, Phys. Rev. B 62, 12569 (2000).

${ }^{16}$ F. Grandjean, G. J. Long, B. Mahieu, D. T. Morelli, J. Yang, and G. P. Meisner, Phys. Rev. B (submitted).

${ }^{17}$ B. C. Sales, B. C. Chakoumakos, and D. Mandrus, Phys. Rev. B 61, 2475 (2000).

${ }^{18}$ A. L. Ankudinov, B. Ravel, J. J. Rehr, and S. D. Conradson, Phys. Rev. B 58, 7565 (1998).

${ }^{19}$ J. J. Rehr and R. C. Albers, Rev. Mod. Phys. 72, 621 (2000).

${ }^{20}$ T. A. Tumolillo, Phys. Status Solidi A 17, 315 (1973).

${ }^{21}$ A. Leithe-Jasper, D. Kaczorowski, P. Rogl, J. Bogner, M. Reissner, W. Steiner, G. Wiesinger, and C. Godart, Solid State Commun. 109, 395 (1999).

${ }^{22}$ F. Grandjean, R. P. Hermann, V. Kuznetsov, and G. J. Long (unpublished).

${ }^{23}$ D. Hautot, G. J. Long, F. Grandjean, and O. Isnard, Phys. Rev. B 62, 11731 (2000).

${ }^{24}$ G. J. Long, D. Hautot, F. Grandjean, O. Isnard, and S. Miraglia, J. Magn. Magn. Mater. 202, 100 (1999).

${ }^{25}$ L. Gelato, J. Appl. Crystallogr. 14, 141 (1981).

${ }^{26}$ G. J. Long, F. Grandjean, B. Mahieu, G. Nolas, and S. Schujman, (unpublished).

${ }^{27}$ P. A. Flinn, in Mössbauer Isomer Shifts, edited by G. K. Shenoy and F. E. Wagner (North Holland, Amsterdam, 1978), p. 593.

${ }^{28}$ A. Kjekshus, D. G. Nicholson, and T. Rakke, Acta Chem. Scand. 27, 1315 (1973).

${ }^{29}$ I. Lefebvre-Devos, M. Lasalle, X. Wallart, J. Olivier-Fourcade, L. Monconduit, and J. C. Jumas, Phys. Rev. B 63, 125110 (2001).

${ }^{30}$ Mössbauer Effect Data Index, edited by J. G. Stevens and V. E. Stevens, (Plenum, New York, 1975), p. 130.

${ }^{31}$ Mössbauer Effect Data Index, edited by J. G. Stevens and V. E. Stevens, (Plenum, New York, 1975), p. 136. 